\title{
Reversal of oral anticoagulation in patients with acute intracerebral hemorrhage
}

\author{
Joji B. Kuramatsu* (D), Jochen A. Sembill and Hagen B. Huttner
}

\begin{abstract}
In light of an aging population with increased cardiovascular comorbidity, the use of oral anticoagulation (OAC) is steadily expanding. A variety of pharmacological alternatives to vitamin K antagonists (VKA) have emerged over recent years (direct oral anticoagulants, DOAC, i.e., dabigatran, rivaroxaban, apixaban, and edoxaban) which show a reduced risk for the occurrence of intracerebral hemorrhage $(\mathrm{ICH})$. Yet, in the event of $\mathrm{ICH}$ under $\mathrm{OAC}(\mathrm{OAC}-\mathrm{ICH})$, hematoma characteristics are similarly severe and clinical outcomes likewise substantially limited in both patients with VKA- and DOAC-ICH, which is why optimal acute hemostatic treatment in all OAC-ICH needs to be guaranteed. Currently, International Guidelines for the hemostatic management of patients with $\mathrm{OAC}-\mathrm{ICH}$ are updated as several relevant large-sized observational studies and recent trials have established treatment approaches for both VKA- and DOAC-ICH. While the management of $\mathrm{VKA}-\mathrm{ICH}$ is mainly based on the immediate reversal of elevated levels of international normalized ratio using prothrombin complex concentrates, hemostatic management of DOAC-associated ICH is challenging requiring specific antidotes, notably idarucizumab and andexanet alfa. This review will provide an overview of the latest studies and trials on hemostatic reversal agents and timing and summarizes the effects on hemorrhage progression and clinical outcomes in patients with OAC-ICH.
\end{abstract}

Keywords: Intracerebral hemorrhage, Anticoagulation reversal, Tranexamic acid, Ciraparantag, Desmopressin

\section{Introduction}

Of all stroke sub-types, intracerebral hemorrhage (ICH) constitutes roughly $15 \%$ and is associated with the worst prognosis [1-3]. Mortality amounts up to $50 \%$ after 1 year, and over two thirds of patients survive with significant functional dependency [3, 4]. Over the last years, randomized controlled trials investigating treatment options to influence functional outcome in general $\mathrm{ICH}$ populations have failed to provide effective treatment strategies [5-9]. Worldwide, the incidence of intracerebral hemorrhage $(\mathrm{ICH})$ is increasing and primarily driven by low- and middle-income countries [1]. Alongside the demographic change in Western Hemisphere countries, and increasing comorbidity requiring oral anticoagulation $(\mathrm{OAC})$, OAC-associated $\mathrm{ICH}$ represents a growing dilemma [10].

Compared to general ICH cohorts, patients with OAC-ICH are older, exhibit larger ICH-volumes, have more frequent intraventricular hemorrhage (IVH), and

\footnotetext{
* Correspondence: joji.kuramatsu@uk-erlangen.de

Department of Neurology, University Hospital Erlangen, Schwabachanlage 6, 91054 Erlangen, Germany
}

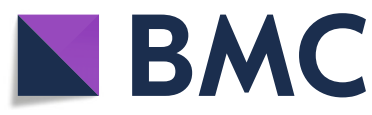

(c) The Author(s). 2019 Open Access This article is distributed under the terms of the Creative Commons Attribution 4.0 International License (http://creativecommons.org/licenses/by/4.0/), which permits unrestricted use, distribution, and

reproduction in any medium, provided you give appropriate credit to the original author(s) and the source, provide a link to the Creative Commons license, and indicate if changes were made. The Creative Commons Public Domain Dedication waiver (http://creativecommons.org/publicdomain/zero/1.0/) applies to the data made available in this article, unless otherwise stated. importantly have a greater frequency of hematoma expansion (HE), all of which are significant outcome predictors determining an even poorer prognosis [11-13]. In vitamin $\mathrm{K}$ antagonist (VKA)-associated $\mathrm{ICH}$, the rate of $\mathrm{HE}$ is described to occur in more than one out of three patients although international normalized ratio (INR) levels were not elevated beyond the therapeutic range. Further, HE may occur protractedly even exceeding $24 \mathrm{~h}$, if anticoagulation status is not reversed [1113]. Comparing $\mathrm{ICH}$ occurring under use of direct oral anticoagulants (DOAC) versus VKA provides at least similar characteristics regarding validated $\mathrm{ICH}$-specific outcome predictors (neurological status, ICH-volume, IVH, HE rates) and clinical outcomes [14-17]. Hence, aggressive and specific medical management to reverse altered coagulation irrespective of anticoagulant used is essential to reduce HE rates and thereby to potentially influence clinical outcomes [18].

\section{Vitamin $\mathrm{K}$ antagonists}

Over the decades, VKA have been the mainstay for anticoagulant treatment in patients with atrial fibrillation 
(A-fib) [19]. A dose-response relationship between bleeding complications and supra-therapeutic international normalized ratio (INR) levels has been documented with a sharp incidence increase for INR levels above 4 [20]. Nevertheless, ICH occurs commonly at therapeutic INR levels exemplified by the largest available VKA-associated ICH cohort $(n=1176)$ reporting a median INR level of 2.8 with an interquartile range between 2.3 and 3.5 [11]. While DOACs are currently replacing VKA as the primary drug in A-fib-patients, VKA remains the primary anticoagulant for several patient populations with high thromboembolic risk [21, 22]. Current American and International Guidelines for the management of $\mathrm{ICH}$ patients do not provide specific recommendations on how to treat VKA-associated $\mathrm{ICH}$ in terms of a specific reversal strategy [3, 23]. Particularly, INR values representing full reversal, timing of reversal, and reversal agents to be used are not addressed. However, guidelines are currently being revised as several high-quality publications have substantially added knowledge to the field.

In 2015, a large observational multicenter study $(n=$ 1176 patients with VKA-ICH) conducted across 19 tertiary care centers in Germany addressed the questions which INR levels should be achieved to most effectively minimize HE [11]. Results showed (for 853 patients with detailed follow-up imaging) that an INR of less than 1.3 was necessary to reduce the risk of $\mathrm{HE}$ (INR $<1.3$, HE rate $=27 \%$, versus INR $>1.3$, HE rate $=45 \%$ ). This association was stronger than the earlier achieved and significantly present until $4 \mathrm{~h}$ after hospital admission (achieved INR $<1.3$ within $4 \mathrm{~h}, \mathrm{HE}$ rate $=20 \%$ versus not achieved HE rate $=42 \%$ ). Notably, these data resulted from a patient population that arrived roughly at a median of $2 \mathrm{~h}$ after symptom onset which is an important factor to be considered as the risk for HE is greatest during the hyper-acute time window $(<3 \mathrm{~h})$, recently verified by a large $(n=5435)$ individual patient data metaanalysis [24]. Therefore, it appears that earlier treatment may translate into a greater effect size for HE prevention. These large-sized investigations strongly support that immediate as well as complete reversal is essential to minimize HE in VKA-ICH, which has also been demonstrated in patients requiring more intense anticoagulation, i.e., mechanical heart valves [22]. In addition to specific reversal treatment, the German multicenter study suggested that lowering of systolic blood pressure below $160 \mathrm{mmHg}$ provided further reductions of HE risk and beyond sole imaging findings significantly reduced in-hospital mortality (odds ratio (OR), 0.6, 95\%CI 0.4$0.9)$ [11]. In this regard, for general $\mathrm{ICH}$ populations, a meta-analysis of five randomized controlled trials $(n=$ 4360) investigating associations of an intensive blood pressure lowering regime (targeting a systolic blood pressure level $<140 \mathrm{mmHg}$ ) versus standard blood pressure lowering (systolic blood pressure level <180 $\mathrm{mmHg}$ ) showed promising results for reduced HE rates (OR 0.2; 95\% CI 0.7 to $1.0, p=0.06$ ) and for death or dependency rates at 3 months (OR $0.9 ; 95 \% \mathrm{CI} 0.8$ to $1.0, p=$ $0.11)$. The current AHA guideline for the management of ICH patients recommends to target systolic RR levels of $140 \mathrm{mmHg}$ during the acute phase of $\mathrm{ICH}[3,25]$.

Agents to be used for reversal treatment have been investigated more thoroughly in general populations with VKA-associated major hemorrhages or acute surgical indications. One randomized phase IIIb, multicenter, open-label, non-inferiority trial, in 202 patients with VKA-associated hemorrhage (only 24 ICH-patients), showed that abnormal coagulation was more rapidly reversed by vitamin $\mathrm{K}$ and 4-factor PCC (containing coagulation factors II, VII, IX, X) applied using staggered

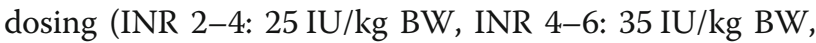
INR > 6: $50 \mathrm{IU} / \mathrm{kg} \mathrm{BW)}$ compared to fresh frozen plasma (FFP dosing, INR 2-4: $10 \mathrm{ml} / \mathrm{kg}$, INR 4-6: $12 \mathrm{ml} / \mathrm{kg}$, INR $>6: 15 \mathrm{ml} / \mathrm{kg}$ ), i.e., INR $\leq 1.3$, achieved by $\mathrm{PCC}+$ vitamin $\mathrm{K}$ in $62.2 \%$ versus FFP + vitamin $\mathrm{K}$ in 9.6\% [26]. In another phase 3 study $(n=181)$, patients with an INR $\geq 2.0$ and acute indication for surgery using the same dosing approach were randomized to receive either 4-factor PCC at or FFP each combined with vitamin K. In the PCC group, surgery could be started earlier after infusion in the PPC group (median $3.6 \mathrm{~h}$ with interquartile range (IQR) 1.9-10.8) compared to the FFP group (median 8.5 IQR, $2.8-18.7 ; p=0.01$ ) and the primary outcome of effective hemostasis was achieved more frequently with PCC (PCC 90\% versus FF 75\%; $p=0.01$ ) [27]. Both trials did show no signals regarding an unfavorable safety profile of PCC. In patients with $\mathrm{ICH}$, the randomized controlled INCH trial included patients with VKA-associated ICH and INR levels greater or equal to 2 on hospital admission to compare 4-factor PCC (30 $\mathrm{IU} / \mathrm{kg} \mathrm{BW})$ versus FFP $(20 \mathrm{ml} / \mathrm{kg} \mathrm{BW})$ both combined with intravenous vitamin $\mathrm{K}(10 \mathrm{mg})$. The primary endpoint was the proportion of patients achieving an INR $\leq$ 1.2 within $3 \mathrm{~h}$, and the trial was prematurely stopped after enrolment of 54 patients as HE rates significantly differed between treatments [28]. A significantly larger proportion of PCC-treated patients (67\%) compared with FFP (9\%) reached the target INR of $\leq 1.2$ after $3 \mathrm{~h}$ (adj. odds ratio 30.6, 95\% CI 4.7-197.9; $p=0.0003$ ). After $24 \mathrm{~h}$, patients in the FFP treatment group had greater increase in ICH volume (absolute ICH volume difference $16.4 \mathrm{~mL}, 95 \%$ CI 2.9-29.9, $p=0.02$ ) and had a fivefold increased risk for $\mathrm{HE}$, defined as a $\mathrm{ICH}$ volume increase of greater $33 \%$ from initial to follow-up imaging (odds ratio $4.5,95 \%$ CI 1.3-20.4, $p=0.02$ ). Although there was a strong trend towards reduced 90-day mortality (PCC, $19 \%$ versus FFP, 35\%), it did not reach statistical 
significance likely related to the small sample size [28]. Importantly, reversal in VKA-associated ICH should be accompanied by simultaneous administration of slow (15-30 min) intravenous infusion of $10 \mathrm{mg}$ vitamin $\mathrm{K}$ (25 ml normal saline) to restore intrinsic hepatic carboxylation of clotting factors and to achieve prolonged hemostasis [29, 30]. Taken together, there is convincing evidence to recommend immediate reversal in VKAassociated ICH to INR levels $<1.3$ as fast as possible and to favor 4-factor PCC over plasma to influence HE rates and clinical outcomes [31].

Management of VKA-associated ICH:

- Immediate INR reversal using 4-factor PCC (25-50 $\mathrm{IU} / \mathrm{kg} \mathrm{BW})$ and vitamin $\mathrm{K}(10 \mathrm{mg})$

- Targeting complete reversal INR $<1.3$ as soon as possible, at least within $4 \mathrm{~h}$

- Timely and serial INR measurements within the acute phase

- Intensive systolic blood pressure reduction, targeting $140 \mathrm{mmHg}$

- Avoid hypotension, i.e., systolic blood pressure level below $100-120 \mathrm{mmHg}$

\section{Direct oral anticoagulants}

Currently marketed non-VKA anticoagulants comprise three factor-Xa inhibitors (apixaban, edoxaban, rivaroxaban, inhibition of the conversion of prothrombin to thrombin) and the direct thrombin-inhibitor dabigatran (competitive inhibitor of thrombin, thereby inhibiting fibrin production), all of which are now recommended for primary or secondary stroke prevention in patients with atrial fibrillation over VKA [19]. Compared to VKA these DOACs share similar pharmacokinetic properties such that elimination half-life is short ranging from 6 to $17 \mathrm{~h}$ across agents in patients with normal renal function [32]. Therefore, effective OAC may theoretically not be present on admission, but importantly cannot be timely and validly excluded by routine diagnostics [33, 34]. Using conventional coagulation testing does not provide sufficient sensitivity or specificity, and currently, no data is available suggesting a definite threshold for all DOACs below which one can exclude DOAC effect [33]. For a rough qualitative estimate of altered hemostasis in DOAC-treated patients in general, thrombin time (TT), prothrombin time (PT), and/or activated partial thromboplastin time may be used. More specifically, if available, quantitative assessment (time-consuming, roughly $30 \mathrm{~min}$ ) of dabigatran levels may be achieved with the dilute thrombin time (dTT), ecarin clotting time (ECT), and for apixaban, edoxaban, and rivaroxaban with agent-specific anti-factor Xa levels, further point-of-care testing devices are currently under development or evaluation [33, 34]. Hence, patients with known DOAC intake and $\mathrm{ICH}$ should all receive immediate reversal treatment. Timing of last DOAC intake is important as early treatment ( $2-4 \mathrm{~h}$ after ingestion) with active charcoal $(50 \mathrm{~g})$, if safely tolerable by the patient, may have the potential to reduce drug absorption [35]. Other more general options may theoretically comprise hemodialysis in dabigatran-related bleedings, but this seems not to be a sensible option in ICH where immediate reversal must be achieved. Currently, DOAC reversal comprises specific and unspecific approaches which will be highlighted in the following.

\section{Specific reversal antidotes}

In DOAC-associated major hemorrhages or patients requiring emergency surgery, several prospective, multicenter, open-label studies investigating the effectiveness of reversal agents are available [36, 37]. Yet, specific analyses of patients with $\mathrm{ICH}$ from these studies are not published yet. Reversal agents differ regarding pharmaco- mechanistic and kinetic properties as well as effectiveness across the various DOAC agents. Therefore, at the current stage, there is no evidence regarding the effectiveness of reversal agents to influence HE rates or clinical endpoints in DOAC-associated ICH.

\section{Ciraparantag for DOAC-associated ICH}

Ciraparantag (syn.: PER977, aripazine), a small molecule $(520 \mathrm{Da})$, was designed to reverse anticoagulatory effect of heparinoids, direct thrombin, and factor-Xa inhibitors and is currently investigated in phase II trials (NCT03172910, NCT03288454). The broad application seems to be an advantage, especially as rapid onset of activity, single-dose application, and long duration of effect have been suggested [38]. A recent phase I/II investigation in 82 healthy male subjects has reported that anticoagulation of edoxaban $(60 \mathrm{mg})$ was reversed within 10 $30 \mathrm{~min}$ as well as over $24 \mathrm{~h}$ by a single-dose ciraparantag (100-300 mg), without increasing procoagulant measures (d-dimer, prothrombin fragments 1.2, and tissue factor pathway inhibitor levels) [39]. Ciraparantag received fast-track designation in 2015; however, currently, it remains uncertain whether or when this agent will be further evaluated in controlled trials targeting FDA approval and market release.

\section{Idarucizumab for dabigatran-associated ICH}

Specific reversal of dabigatran can be achieved with idarucizumab, which is a non-competitive inhibitor and represents a humanized monoclonal antibody fragment binding to dabigatran with high affinity (350 times greater than thrombin). The formation of this complex between idarucizumab and dabigatran is almost irreversible; hence, anticoagulation persistently reversed, and this complex renally excreted [36, 40]. Idarucizumab 
(Praxbind ${ }^{\circ}$ ) is administered as two intravenous boluses $(2 \times 2.5 \mathrm{~g})$ within $15 \mathrm{~min}$ and gained approval by the European Medicines Agency (EMA) and Federal Drug Administration (FDA) in 2015 for reversal of dabigatranassociated life-threating bleeding complications or for patients requiring emergency surgery.

The full-cohort analyses of the open-label REVERSEAD study was published in 2017, comprised overall 503 patients grouped into patients with uncontrolled hemorrhage (group A, $n=301$ ) or in need for urgent invasive procedures (group $\mathrm{B}, n=202$ ) [36]. The primary endpoint consisted of the maximum percentage reversal of the anticoagulant effect, measured by the dTT or ecarin-clotting time within the first $4 \mathrm{~h}$ after infusion of idarucizumab. Results provided for the entire cohort that on admission $92 \%$ of patients had prolonged bleeding measures and $4 \mathrm{~h}$ after reversal treatment the median maximum percentage reversal was $100 \%$ [36]. For group A, including 98 patients with intracranial hemorrhage of which 53 patients experienced $\mathrm{ICH}$, the median level of unbound dabigatran was initially $110 \mathrm{ng} / \mathrm{ml}$ and after reversal was $20 \mathrm{ng} / \mathrm{ml}$ and remained below this level for $24 \mathrm{~h}$ suggesting impairment of anticoagulation to be very unlikely. In patients with intracranial hemorrhage, protocolized follow-up imaging was not mandated; therefore, effects on HE rates cannot be reported. In patients with gastrointestinal hemorrhage $(n=137)$, further clinical assessment showed that median time to bleeding cessation was $2.5 \mathrm{~h}$. For the entire cohort, the reported thromboembolic event rate was 5\% (24/503) within 30 days and in patients with $\mathrm{ICH} 6 \%(3 / 53)$ experienced a thromboembolic event, all occurring more than 10 days after idarucizumab administration [36].

Mechanistically, idarucizumab is not expected to generate an intrinsic prothrombotic risk, and the reported events are likely to be associated with the underlying disease. Data specifically in $\mathrm{ICH}$ patients is very limited, and smaller case series based on a prospective German nation-wide observational study reported $\mathrm{HE}$ rate of $25 \%(2 / 8)$ after reversal with idarucizumab theoretically reflecting a $\mathrm{HE}$ rate comparable to VKA-associated $\mathrm{ICH}$ patients being reversed to INR levels below 1.3 [41]. Even though randomized data and detailed analysis of $\mathrm{ICH}$ patients is lacking, findings of the REVERSE-AD study for dabigatran-associated ICH suggest that the specific antidote idarucizumab provides a rapid, sufficient, and prolonged reversal of anticoagulation effect and should be immediately administered after diagnosis of ICH as two intravenous boluses $(2 \times 2.5 \mathrm{~g})$ within 15 $\min [36]$.

\section{Andexanet alfa for factor-Xa-inhibitor-associated ICH}

Specific reversal of factor-Xa-inhibitors (rivaroxaban, apixaban) can be accomplished with andexanet alfa
(Andexxa $^{\circ}$ ) which has been approved in May 2018 by the FDA. Andexanet alfa has been designed to reverse the anticoagulant activity of both direct and indirect factor-Xa-inhibitors [42]. Andexanet alfa acts as a human decoy receptor binding to the active site of factorXa inhibitors with high affinity and possesses no catalytic activity [42]. Therefore, factor-Xa activity is supposedly restored and effect of anticoagulation attenuated. Several studies in healthy subjects have been conducted to evaluate the potential of reversing anticoagulation. More specifically, a two-part randomized controlled phase 3 trial (ANNEXA-A and ANNEXA-R) evaluated effectiveness of andexanet alfa in healthy older volunteers taking either apixaban (ANNEXA-A, $n=24$ ) or rivaroxaban (ANNEXA-R, $n=27$ ) comparing different dosing (400-960 mg) and application regimes (single bolus and bolus followed by 2-h infusion) [43]. Study results showed that in over $90 \%$ anti-Xa activity was reduced during time of treatment with andexanet alfa followed by a rebound after end of infusion [43].

Currently, the phase 4 study (NCT02329327) is ongoing, and most recently, the full study report has been published [37]. For this multicenter, prospective, openlabel, single-group study patients with factor-Xa inhibitor (apixaban, edoxaban, rivaroxaban, and enoxaparin) associated hemorrhages within $18 \mathrm{~h}$ after the last intake has been published. The coprimary outcomes were the percent change in anti-factor-Xa activity after andexanet treatment and the percentage of patients with excellent or good hemostatic efficacy at $12 \mathrm{~h}$ after the end of the infusion, as pre-specified [37]. The treatment protocol comprised a bolus infusion over 15 to $30 \mathrm{~min}$ followed by a 2-h infusion, with different dosing dichotomized according to last known intake, i.e., intake within the last $7 \mathrm{~h}$ or unknown status received a higher dose of $800 \mathrm{mg}$ over $30 \mathrm{~min}$ followed by $960 \mathrm{mg}$, and last intake $>7 \mathrm{~h}$ received $400 \mathrm{mg}$ followed by $480 \mathrm{mg}$. Included patients $(n=352)$ exhibited dominantly intracranial hemorrhages in $64 \%(n=227 / 352)$, including $241 \mathrm{ICH}$ patients, and $20 \%$ with gastrointestinal hemorrhages [37]. By study design efficacy, analysis was conducted for 254 patients and results showed a decrease in anti-factor-Xa activity in over $90 \%$ in apixaban and rivaroxaban and in $75 \%$ of enoxaparin-treated patients measured 4,8 , and $12 \mathrm{~h}$ after infusion. Predefined excellent or good hemostasis assessed $12 \mathrm{~h}$ after the end of infusion was achieved in $82 \%$ (95\% CI 77-87\%) of patients.

Specifically, focusing on patients with $\mathrm{ICH}$, study results are not published but have been reported at the International Stroke Conference 2019. In $71 \mathrm{ICH}$ patients eligible for efficacy analyses, HE was reported in 15 patients assessed at $1 \mathrm{~h}$ and in 1 patient assessed at $12 \mathrm{~h}$. Therefore, the HE rate can be considered to be at $22 \%(n=16 / 71)$ in ICH patients after andexanet 
infusion, theoretically again comparable to dabigatranassociated and VKA-associated ICH patients receiving reversal treatment. Interestingly, for the entire cohort, no correlation between hemostatic efficacy and decreased anti-factor-Xa activity was noted, but in ICH patients, a moderate correlation could be shown as area under the curve of $0.64,95 \% \mathrm{CI}(53-74)$. A debated matter of concern for this study was the reported thrombotic event rate of $10 \%(n=34 / 352)$ including $4 \%(n=$ $15)$ with ischemic stroke and $4 \%(n=13 \%)$ with deep vein thrombosis in light of elevated laboratory surrogates (d-dimer, prothrombin fragments 1 and 2). ANNEXA-4 is still ongoing to further evaluate also patients with edoxaban associated hemorrhages and for more detailed analyses of $\mathrm{ICH}$ patients. However, comparing the estimated treatment cost (based on US data) of reversal agents suggests a large discrepancy between idarucizumab (5495 USD) or 4-factor PCC (4000 IU, 5080 USD) and andexanet alfa ranging between 24,000 and 48,000 USD [32, 44]. So far, andexanet alfa is only approved in the USA. The European Medicines Agency (EMA) has agreed to consider andexanet alfa (Ondexxya ${ }^{\circ}$ ) for fast track approval provided results of the ongoing trial. Outside the USA, andexanet alfa can (theoretically already now) be purchased through international pharmacies at extremely expensive costs.

In summary, for factor-Xa inhibitor-related $\mathrm{ICH}$, through providing fast and sufficient effect on hemostasis, andexanet alfa harbors several limitations which make administration more complex as compared to idarucizumab. The hemostatic rebound, the need for continuous infusion, the reported prothrombotic complications, and the financial aspects bare the risk that andexanet alfa will ultimately not be used as frequently as necessary. In addition, similar to idarucizumab, data on HE rates and clinical outcomes are necessary in order to verify clinical relevance of andexanet alfa in $\mathrm{ICH}$ patients [36, 37].

\section{Unspecific reversal approaches PCC for factor-Xa inhibitor-associated ICH}

Three categories of human plasma compounds-prothrombin complex concentrates (PCC)-are currently available to restore altered coagulation, i.e., 3-factor PCC (II, IX, X), 4-factor PCC (II, VII, IX, X), and activated PCC (activated VII, II, IX, X, FEIBA) [34]. Experimental data and mostly phase I randomized data have suggested that PCC may have potential to reverse anticoagulation induced by factor-Xa inhibitors [45-48]. For direct comparisons of these factor concentrates, most data is present for anticoagulation treatment with rivaroxaban and edoxaban suggesting effects of 4-factor PCC over 3factor PCC and FEIBA being comparable if not superior to 4-factor PCC to reverse coagulation. A small cross- over study in 10 healthy volunteers, treated with dabigatran and rivaroxaban, suggested that thrombin generation was improved best by activated 4-factor PCC measured by ex vivo hemostatic testing of PCC derivatives in rivaroxaban-treated but not in dabigatrantreated patients [46]. Direct human in vivo comparisons between FEIBA and 4-factor PCC are not available. Clinically more convincing are data investigated in 35 healthy individuals receiving PCC, comparing 3-factor with 4-factor PCC, which showed for both agents possible reversal properties after rivaroxaban treatment [47]. The largest randomized study was conducted in 110 healthy edoxaban-treated (single dose $60 \mathrm{mg}$ ) individuals and compared dosing regimens using 4-factor PCC (50 $\mathrm{IU} / \mathrm{kg} \mathrm{BW}, 25 \mathrm{IU} / \mathrm{kg} \mathrm{BW}, 10 \mathrm{IU} / \mathrm{kg} \mathrm{BW}$ ) and determined effect based on bleeding duration and volume after dermal punch biopsy. Results provided that PCC administered only at $50 \mathrm{IU}$ per $\mathrm{kg}$ body weight influenced both bleeding endpoints supporting a potential role for in unspecific reversal [48]. No adverse events occurred that were adjudicated to be related to the study medication.

In patients with major hemorrhagic complications and/or ICH under use of rivaroxaban or apixaban, a prospective cohort study $(n=84)$ including 59 patients with intracranial hemorrhage investigated associations of 4factor PCC with hemostasis rate, as defined per study protocol [49]. For the entire cohort, the median PCC dose was $2000 \mathrm{IU}$ (IQR $1500-2000$ ) or $27 \mathrm{IU} / \mathrm{kg} \mathrm{BW}$ and "effective" hemostasis was scored in $69 \%(n=58 / 84)$ of patients. For patients with intracranial hemorrhage, ineffective hemostasis was reported in $27 \%$ (16/59), conferring to similar HE rates as available for reversal with andexanet or idarucizumab [49]. The thromboembolic rate however was rather low with $4 \%(3 / 84)$. One of the first larger observational studies $(n=61)$ in ICH patients did not show signals that PCC was influencing HE rates (43\% $n=12 / 28$ received PCC versus $29 \% n=5 / 17$ without PCC, $p=0.5)$ [17]. The largest available cohort study ( $n=190$; rivaroxaban, $n=142$; apixaban, $n=26$; dabigatran, $n=22$ ) was based on the follow-up study (2010-2015) from the German-wide multicenter study (RETRACE-program) and, according to study protocol, included only patients with ICH under known DOAC use [14]. For the differing DOAC agents observed HE rates in patients with detailed follow-up imaging were $33 \%$ for rivaroxaban, $48 \%$ for apixaban, and $20 \%$ for dabigatran, which were not statistically different, but larger sample sizes would be required to determine potential DOAC class associations. Specific analyses of HE rates according to reversal strategies provided that across all agents the median PCC dose given was 2000 IU for rivaroxaban (IQR 1500-2600) and dabigatran (IQR 1650-3000) and 2400 IU for apixaban (IQR 15003000). But it has to be recognized that overall less than 
half of all patients received appropriate dosing (dose $\geq$ $25 \mathrm{IU} / \mathrm{kg} \mathrm{BW}$; 44\%, $n=65 / 146)$ which was recommended during that treatment period.

Current consensus recommendations support higher dosing with $50 \mathrm{IU} / \mathrm{kg} \mathrm{BW}$ or greater, and within that study, only $5 \%$ of patients were treated accordingly; therefore, sensible analyses of this higher-dosed regime was not executable. Upon adjusted analyses, this multicenter study provided no effect of PCC reversal on reduction of $\mathrm{HE}$ rates in factor-Xa inhibitor-associated ICH (risk ratio $1.06,95 \% \mathrm{CI} 0.56-1.98$ ) or on clinical endpoints. However, it is always important to identify patients with high re-bleeding risk in whom aggressive medical treatment possesses greater effect size. For rivaroxaban specific anti-Xa-activity levels of greater of 118 $\mathrm{ng} / \mathrm{ml}$ were identified to be significantly associated with increased HE risk (level $>118 \mathrm{ng} / \mathrm{ml}$, HE rate $56 \%$ versus level $\leq 118 \mathrm{ng} / \mathrm{ml}$, HE rate $17 \%$; $p=0.01$ ). Upon further categorizing sub-group analyses, no other association, but systolic blood pressure reduction $(<160 \mathrm{mmHg}$, risk ratio $0.6,95 \% \mathrm{CI}(0.36-0.98), p=0.04)$, could be identified as protective on HE. Therefore, available data suggests that 4-factor PCC at a dose of $50 \mathrm{IU} / \mathrm{kg}$ BW may be considered as "second-line" treatment in factor-Xainhibitor-associated $\mathrm{ICH}$, if andexanet is unavailable.

\section{Other hemostatic agents}

Recently, a large randomized trial $(n=2325)$ investigated the effect of the anti-fibrinolytic agent tranexamic acid ( $1 \mathrm{~g}$ Bolus, followed by $1 \mathrm{~g}$ infusion over $8 \mathrm{~h}$ ) on functional outcome after 90 days in patients with primary $\mathrm{ICH}$, but per study protocol excluded patients with OAC [6]. Results provided no significant effect on functional outcome, yet sub-analyses suggested a significant relation with reduced $\mathrm{HE}$ ( $\mathrm{ICH}$ volume $>33 \%$; binary odds ratio $0.8,95 \% \mathrm{CI}(0.66-0.98), p=0.03)$. Nevertheless, clinical data on associations of tranexamic acid in OACassociated hemorrhage are sparse. The large international trial $(n=20.211)$, Effects of tranexamic acid on death, vascular occlusive events, and blood transfusion in trauma patients with significant haemorrhage (CRASH2), showed a significant risk reduction for bleedingassociated death (relative risk $0.85,95 \%$ CI (0.76-0.96); $p=0.008)$ and did not exclude anticoagulated patients as the study protocol incorporated an uncertainty principle, but OAC use was unlikely in this fairly young (mean age 35 years) study population [50]. Post hoc analyses in traumatic brain injury also provided a decrease of intracranial hemorrhage progression but data on association with OAC are not present [51], but may possibly be generated from the follow-up trial CRASH-3 in traumatic brain injury (NCT01402882). Nevertheless, a smaller multicenter RCT is currently enrolling patients to evaluate tranexamic acid application in DOAC-associated
ICH (TICH-NOAC, NCT02866838), yet available experimental data does not support this hypothesis [52]. Following the negative results and safety concerns with an increased rate of thromboembolic complications in the FAST Trial, a phase 3 study on the efficacy of recombinant activated factor VII (rFVIIa) in ICH patients, administration of rFVIIa is currently not recommended [53], yet ex vivo and in vitro studies suggest reversal effects of rivaroxaban and apixaban by recombinant FVIIa [54]. Another consideration refers to patients under dual therapy-OAC and concomitant antiplatelet medication-which is present in roughly $10 \%$ of patients $(n=$ $290 / 2504$ ) possibly necessitating adjunctive therapies such as platelet transfusions or desmopressin (DDAVP) treatment [55]. For treatment with platelet transfusions, randomized phase 3 trial data $(n=190)$ in antiplateletassociated ICH suggests a negative association with functional outcome at 3 months (adjusted common odds ratio $2.05,95 \%$ CI $(1.18-3.56) ; p=0.01)$ and increased severe adverse events (adjusted common odds ratio 1.79, 95\% CI (0.98-3.27)) [5]. Meta-analyses for DDAVP treatment $(0.4 \mathrm{mcg}$ per $\mathrm{kg} \mathrm{BW})$ in patients with platelet dysfunction or with antiplatelet medication support DDAVP use in patients undergoing surgery to reduce bleeding and transfusion requirements [56]. Specifically, in ICH patients, data is very limited but suggests associations with improved platelet activity [30].

Management of DOAC-associated ICH:

- Consider per oral charcoal (50 g), if last intake $<4 \mathrm{~h}$ and safe for the patient

- Intensive systolic blood pressure reduction, targeting $140 \mathrm{mmHg}$

- Avoid hypotension, i.e., systolic blood pressure level below 100-120 mmHg

- Dabigatran-associated ICH, immediate reversal using Idarucizumab $(2 \times 2.5 \mathrm{~g})$

- Factor-Xa inhibitor-associated ICH, immediate specific reversal using andexanet alfa (unknown time window or last intake $\leq 7 \mathrm{~h}, 800 \mathrm{mg}$ over $30 \mathrm{~min}$ followed by $960 \mathrm{mg}$ over $2 \mathrm{~h}$, last intake $>7 \mathrm{~h}, 400$ mg over $15 \mathrm{~min}$ followed by $480 \mathrm{mg}$ over $2 \mathrm{~h}$ )

- Factor-Xa-inhibitor-associated ICH, immediate unspecific reversal using high-dose 4-factor PCC or activated PCC (both $50 \mathrm{IU} / \mathrm{kg} \mathrm{BW)}$

- Consider serial specific coagulation measurement to monitor reversal (dabigatran, dTT, ECT; factor-Xa inhibitors, agent-specific anti-Xa activity).

\section{Conclusions and future directions}

Significant progress has been achieved recently in large studies for the acute management of patients with OAC$\mathrm{ICH}$. All these therapeutic interventions mainly focus on reducing the occurrence and extent of hematoma 


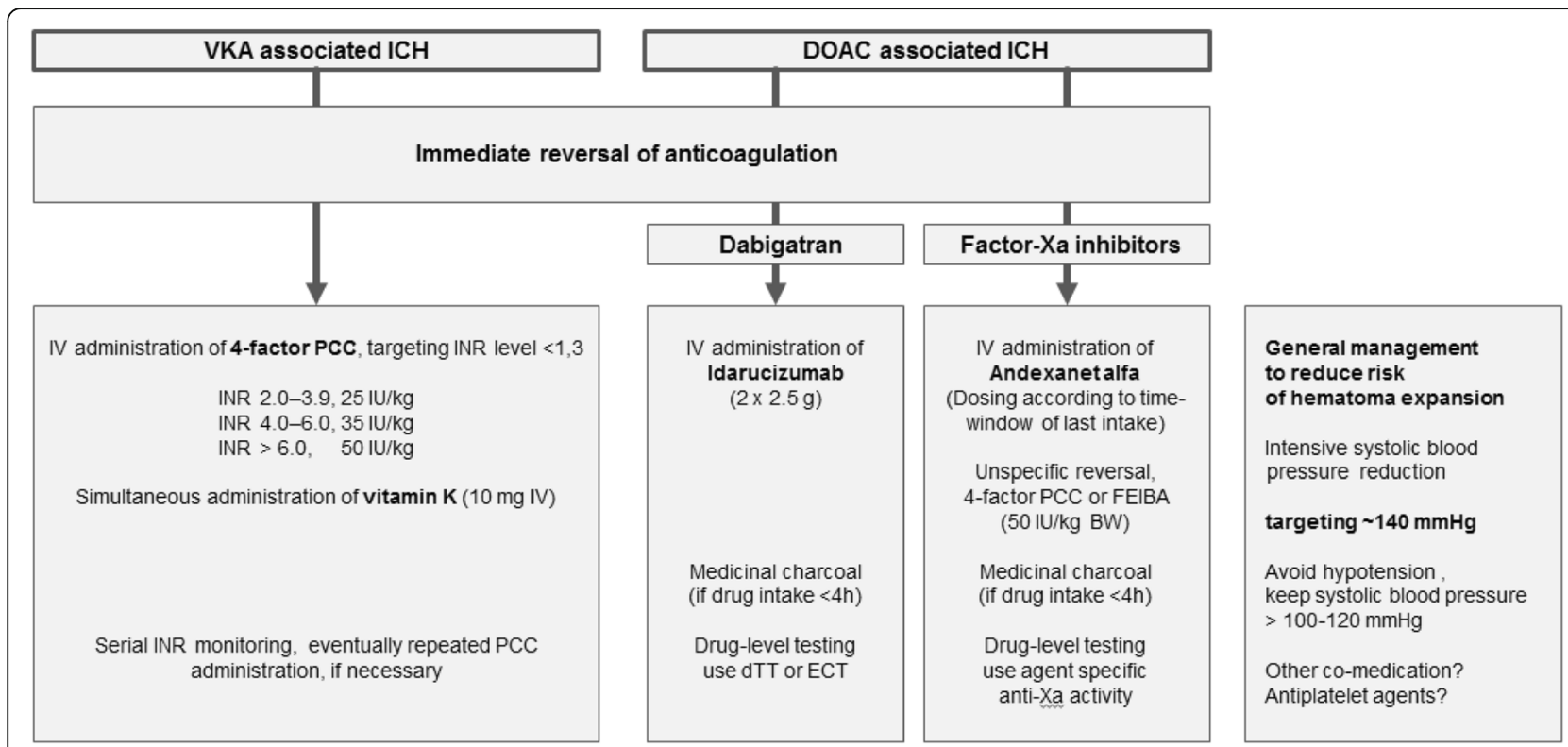

Fig. 1 Acute management of anticoagulation-associated intracerebral hemorrhage. Listed values vary according to renal function and drug interactions. Consult product characteristics for individual decision making. h, hours; ICH, intracerebral hemorrhage; IV, intravenous; DOAC, direct oral anticoagulants; PCC, prothrombin complex concentrate; VKA, vitamin K antagonist. FEIBA, activated 4-factor PCC; IU, international units; kg, kilogram; BW, body weight

enlargement (please see Fig. 1). On the one hand, blood pressure management targeting systolic levels of 140 $\mathrm{mmHg}$ has been verified to limit hematoma expansion and thus should be maintained attentively. On the other hand, optimal hemostatic management significantly restricts hemorrhage progression in all types of OAC-ICH. Specifically, in patients with VKA-ICH, complete reversal of elevated INR levels using prothrombin complex concentrates needs to be initiated immediately to stabilize the intracerebral hematoma. In patients with dabigatran-related $\mathrm{ICH}$, prompt administration of the antidote idarucizumab achieves rapid hemostasis. Although clinical data of minimizing hematoma enlargement by idarucizumab are pending, the likelihood thereof results in a clear recommendation for idarucizumab in dabigatran-ICH. For patients with factor-Xainhibitor-associated $\mathrm{ICH}$, andexanet alfa has been demonstrated to provide sufficient hemostasis and first unpublished data suggest effects on reducing hematoma progression in these patients. However, andexanet alfa currently is approved by the FDA only, whereas it is not available elsewhere in the world, and moreover has not been verified for treatment of all factor-Xa-inhibitors. Hence, hemostatic reversal management for factor-Xainhibitor-associated ICH remains challenging. Although not sufficiently backed by hemostasiological data, and no formally verified safety analysis, International Guidelines recommend prothrombin complex concentrates administration in dosages of $50 \mathrm{IU} / \mathrm{kg}$ bodyweight. Observational analysis on prothrombin complex concentrates administration in factor-Xa-inhibitor-associated ICH showed conflicting data on whether or not there are associations with reduced hemorrhage progression. Two major aspects need to be resolved in timely fashion: firstly, it requires a verification that antidotes, or other prothrombotic drugs respectively, significantly limit hematoma expansion and impact clinical outcomes, and secondly, the all over availability of those drugs needs to be ensured given an increasing worldwide demand.

\section{Abbreviations}

BW: Body weight; Cl: Confidence interval; DOAC: Direct oral anticoagulants; h: Hours; HE: Hematoma expansion; ICH: Intracerebral hemorrhage; INR: International normalized ratio; IU: International units; kg: Kilogram; OAC: Oral anticoagulation; OAC-ICH: Oral anticoagulation-associated intracerebral hemorrhage; VKA: Vitamin $\mathrm{K}$ antagonists

\section{Authors' contributions}

Conception and design, interpretation, and drafting of the work have been conducted by all authors. All authors read and approved the final manuscript.

\section{Funding}

No funding related to the manuscript has been received.

\section{Availability of data and materials Not applicable.}

Ethics approval and consent to participate Not applicable

\section{Consent for publication}

Final approval of the version to be published has been given by all authors. The corresponding has received consent and agreement, to be accountable 
for all aspects of the work in ensuring that questions related to the accuracy or integrity of any part of the work for publication, by all co-authors.

\section{Competing interests}

The authors declare that they have no competing interests.

Received: 20 February 2019 Accepted: 26 May 2019

Published online: 06 June 2019

\section{References}

1. Krishnamurthi RV, Feigin VL, Forouzanfar MH, Mensah GA, Connor M, Bennett DA, et al. Global and regional burden of first-ever ischaemic and haemorrhagic stroke during 1990-2010: findings from the Global Burden of Disease Study 2010. Lancet Glob Health. 2013;1(5):e259-81.

2. Sacco S, Marini C, Toni D, Olivieri L, Carolei A. Incidence and 10-year survival of intracerebral hemorrhage in a population-based registry. Stroke. 2009; 40(2):394-9.

3. Hemphill JC 3rd, Greenberg SM, Anderson CS, Becker K, Bendok BR Cushman $\mathrm{M}$, et al. Guidelines for the management of spontaneous intracerebral hemorrhage: a guideline for healthcare professionals from the American Heart Association/American Stroke Association. Stroke. 2015;46(7): 2032-60.

4. Sembill JA, Gerner ST, Volbers B, Bobinger T, Lucking H, Kloska SP, et al. Severity assessment in maximally treated $\mathrm{ICH}$ patients: the max-ICH score. Neurology. 2017;89(5):423-31.

5. Baharoglu MI, Cordonnier C, Salman RA, de Gans K, Koopman MM, Brand A et al. Platelet transfusion versus standard care after acute stroke due to spontaneous cerebral haemorrhage associated with antiplatelet therapy (PATCH): a randomised, open-label, phase 3 trial. Lancet. 2016;387(10038): 2605-13.

6. Sprigg N, Flaherty K, Appleton JP, Salman RA, Bereczki D, Beridze M, et al. Tranexamic acid for hyperacute primary IntraCerebral Haemorrhage $(\mathrm{TICH}$ 2): an international randomised, placebo-controlled, phase 3 superiority trial. Lancet. 2018;391(10135):2107-115.

7. Hanley DF, Lane K, McBee N, Ziai W, Tuhrim S, Lees KR, et al. Thrombolytic removal of intraventricular haemorrhage in treatment of severe stroke: results of the randomised, multicentre, multiregion, placebo-controlled CLEAR III trial. Lancet. 2017;389(10069):603-11.

8. Hanley DF, Thompson RE, Rosenblum M, Yenokyan G, Lane K, McBee N, et al. Efficacy and safety of minimally invasive surgery with thrombolysis in intracerebral haemorrhage evacuation (MISTIE III): a randomised, controlled, open-label, blinded endpoint phase 3 trial. Lancet. 2019.

9. Mendelow AD, Gregson BA, Rowan EN, Murray GD, Gholkar A, Mitchell PM. Early surgery versus initial conservative treatment in patients with spontaneous supratentorial lobar intracerebral haematomas (STICH II): a randomised trial. Lancet. 2013;382(9890):397-408

10. Kuramatsu JB, Huttner HB. Management of oral anticoagulation after intracerebral hemorrhage. Int J Stroke. 2019;14(3):238-46.

11. Kuramatsu JB, Gerner ST, Schellinger PD, Glahn J, Endres M, Sobesky J, et al. Anticoagulant reversal, blood pressure levels, and anticoagulant resumption in patients with anticoagulation-related intracerebral hemorrhage. JAMA. 2015;313(8):824-36.

12. Flibotte JJ, Hagan N, O'Donnell J, Greenberg SM, Rosand J. Warfarin, hematoma expansion, and outcome of intracerebral hemorrhage. Neurology. 2004;63(6):1059-64.

13. Flaherty ML, Tao H, Haverbusch M, Sekar P, Kleindorfer D, Kissela B, et al. Warfarin use leads to larger intracerebral hematomas. Neurology. 2008; 71(14):1084-9.

14. Gerner ST, Kuramatsu JB, Sembill JA, Sprugel MI, Endres M, Haeusler KG, et al. Association of prothrombin complex concentrate administration and hematoma enlargement in non-vitamin $\mathrm{K}$ antagonist oral anticoagulantrelated intracerebral hemorrhage. Ann Neurol. 2018;83(1):186-96.

15. Inohara T, Xian Y, Liang L, Matsouaka RA, Saver JL, Smith EE, et al. Association of intracerebral hemorrhage among patients taking non-vitamin $\mathrm{K}$ antagonist vs vitamin $\mathrm{K}$ antagonist oral anticoagulants with in-hospital mortality. JAMA. 2018;319(5):463-73.

16. Tsivgoulis G, Wilson D, Katsanos AH, Sargento-Freitas J, Marques-Matos C, Azevedo $\mathrm{E}$, et al. Neuroimaging and clinical outcomes of oral anticoagulantassociated intracerebral hemorrhage. Ann Neurol. 2018;84(5):694-704.

17. Purrucker JC, Haas K, Rizos T, Khan S, Wolf M, Hennerici MG, et al. Early clinical and radiological course, management, and outcome of intracerebral hemorrhage related to new oral anticoagulants. JAMA Neurol. 2016;73(2): 169-77.

18. Sembill JA, Huttner HB, Kuramatsu JB. Impact of recent studies for the treatment of intracerebral hemorrhage. Curr Neurol Neurosci Reports. 2018; 18(10):71.

19. Steffel J, Verhamme P, Potpara TS, Albaladejo P, Antz M, Desteghe L, et al. The 2018 European Heart Rhythm Association Practical Guide on the use of non-vitamin $\mathrm{K}$ antagonist oral anticoagulants in patients with atrial fibrillation. Eur Heart J. 2018;39(16):1330-93.

20. Hylek EM, Go AS, Chang Y, Jensvold NG, Henault LE, Selby JV, et al. Effect of intensity of oral anticoagulation on stroke severity and mortality in atrial fibrillation. N Engl J Med. 2003;349(11):1019-26.

21. Sharma M, Cornelius VR, Patel JP, Davies JG, Molokhia M. Efficacy and harms of direct oral anticoagulants in the elderly for stroke prevention in atrial fibrillation and secondary prevention of venous thromboembolism: systematic review and meta-analysis. Circulation. 2015;132(3):194-204.

22. Kuramatsu JB, Sembill JA, Gerner ST, Sprugel MI, Hagen M, Roeder SS, et al. Management of therapeutic anticoagulation in patients with intracerebral haemorrhage and mechanical heart valves. Eur Heart J. 2018;39(19):1709-23.

23. Steiner T, Al-Shahi Salman R, Beer R, Christensen H, Cordonnier C, Csiba L, et al. European stroke organisation (ESO) guidelines for the management of spontaneous intracerebral hemorrhage. Int J Stroke. 2014;9(7):840-55.

24. Al-Shahi Salman R, Frantzias J, Lee RJ, Lyden PD, Battey TWK, Ayres AM, et al. Absolute risk and predictors of the growth of acute spontaneous intracerebral haemorrhage: a systematic review and meta-analysis of individual patient data. Lancet Neurol. 2018;17(10):885-94.

25. Boulouis G, Morotti A, Goldstein JN, Charidimou A. Intensive blood pressure lowering in patients with acute intracerebral haemorrhage: clinical outcomes and haemorrhage expansion. Systematic review and meta-analysis of randomised trials. J Neurol Neurosurg Psychiatry. 2017:88(4):339-45.

26. Sarode R, Milling TJ Jr, Refaai MA, Mangione A, Schneider A, Durn BL, et al. Efficacy and safety of a 4-factor prothrombin complex concentrate in patients on vitamin $\mathrm{K}$ antagonists presenting with major bleeding: a randomized, plasma-controlled, phase IIlb study. Circulation. 2013;128(11): 1234-43.

27. Goldstein JN, Refaai MA, Milling TJ Jr, Lewis B, Goldberg-Alberts R, Hug BA, et al. Four-factor prothrombin complex concentrate versus plasma for rapid vitamin $\mathrm{K}$ antagonist reversal in patients needing urgent surgical or invasive interventions: a phase 3b, open-label, non-inferiority, randomised trial. Lancet. 2015;385(9982):2077-87.

28. Steiner T, Poli S, Griebe M, Husing J, Hajda J, Freiberger A, et al. Fresh frozen plasma versus prothrombin complex concentrate in patients with intracranial haemorrhage related to vitamin $\mathrm{K}$ antagonists $(\mathrm{INCH})$ : a randomised trial. Lancet Neurol. 2016;15(6):566-73.

29. Watson HG, Baglin T, Laidlaw SL, Makris M, Preston FE. A comparison of the efficacy and rate of response to oral and intravenous vitamin $\mathrm{K}$ in reversal of over-anticoagulation with warfarin. Br J Haematol. 2001;115(1):145-9.

30. Frontera JA, Lewin JJ 3rd, Rabinstein AA, Aisiku IP, Alexandrov AW, Cook AM, et al. Guideline for reversal of antithrombotics in intracranial hemorrhage: a statement for healthcare professionals from the Neurocritical Care Society and Society of Critical Care Medicine. Neurocrit Care. 2016; 24(1):6-46.

31. Chai-Adisaksopha C, Hillis C, Siegal DM, Movilla R, Heddle N, lorio A, et al. Prothrombin complex concentrates versus fresh frozen plasma for warfarin reversal. A systematic review and meta-analysis. Thromb Haemost. 2016; 116(5):879-90.

32. Piran S, Schulman S. Treatment of bleeding complications in patients on anticoagulant therapy. Blood. 2019;133(5):425-35.

33. Douxfils J, Gosselin RC. Laboratory assessment of direct oral anticoagulants. Semin Thromb Hemost. 2017:43(3):277-90.

34. Steiner T, Kohrmann M, Schellinger PD, Tsivgoulis G. Non-vitamin K oral anticoagulants associated bleeding and its antidotes. J Stroke. 2018;20(3):292-301.

35. Wang X, Mondal S, Wang J, Tirucherai G, Zhang D, Boyd RA, et al. Effect of activated charcoal on apixaban pharmacokinetics in healthy subjects. Am J Cardiovasc Drugs. 2014;14(2):147-54.

36. Pollack CV Jr, Reilly PA, van Ryn J, Eikelboom JW, Glund S, Bernstein RA, et al. Idarucizumab for dabigatran reversal - full cohort analysis. N Engl J Med. 2017:377(5):431-41.

37. Connolly SJ, Crowther M, Eikelboom JW, Gibson CM, Curnutte JT, Lawrence $\mathrm{JH}$, et al. Full study report of andexanet alfa for bleeding associated with factor Xa inhibitors. New England J Med 2019. 
38. Ansell JE, Bakhru SH, Laulicht BE, Steiner SS, Grosso M, Brown K, et al. Use of PER977 to reverse the anticoagulant effect of edoxaban. N Engl J Med. 2014;371(22):2141-2.

39. Ansell JE, Bakhru SH, Laulicht BE, Steiner SS, Grosso MA, Brown K, et al. Single-dose ciraparantag safely and completely reverses anticoagulant effects of edoxaban. Thromb Haemost. 2017;117(2):238-45.

40. Pollack CV Jr, Reilly PA, Eikelboom J, Glund S, Verhamme P, Bernstein RA, et al. Idarucizumab for dabigatran reversal. N Engl J Med. 2015;373(6):511-20.

41. Kermer P, Eschenfelder CC, Diener HC, Grond M, Abdalla Y, Althaus K, et al. Antagonizing dabigatran by idarucizumab in cases of ischemic stroke or intracranial hemorrhage in Germany - a national case collection. Int J Stroke. 2017;12(4):383-91.

42. Connolly SJ, Milling TJ Jr, Eikelboom JW, Gibson CM, Curnutte JT, Gold A, et al. Andexanet alfa for acute major bleeding associated with factor Xa inhibitors. N Engl J Med. 2016.

43. Siegal DM, Curnutte JT, Connolly SJ, Lu G, Conley PB, Wiens BL, et al. Andexanet alfa for the reversal of factor Xa inhibitor activity. N Engl J Med. 2015;373(25):2413-24.

44. Bower MM, Sweidan AJ, Shafie M, Atallah S, Groysman LI, Yu W. Contemporary reversal of oral anticoagulation in intracerebral hemorrhage. Stroke. 2019;50(2):529-36.

45. Eerenberg ES, Kamphuisen PW, Sijpkens MK, Meijers JC, Buller HR, Levi M. Reversal of rivaroxaban and dabigatran by prothrombin complex concentrate: a randomized, placebo-controlled, crossover study in healthy subjects. Circulation. 2011;124(14):1573-9.

46. Marlu R, Hodaj E, Paris A, Albaladejo P. Cracowski JL, Pernod G. Effect of non-specific reversal agents on anticoagulant activity of dabigatran and rivaroxaban: a randomised crossover ex vivo study in healthy volunteers. Thromb Haemost. 2012;108(2):217-24.

47. Levi M, Moore KT, Castillejos CF, Kubitza D, Berkowitz SD, Goldhaber SZ, et al. Comparison of three-factor and four-factor prothrombin complex concentrates regarding reversal of the anticoagulant effects of rivaroxaban in healthy volunteers. J Thromb Haemostasis. 2014;12(9):1428-36.

48. Zahir H, Brown KS, Vandell AG, Desai M, Maa JF, Dishy V, et al. Edoxaban effects on bleeding following punch biopsy and reversal by a 4-factor prothrombin complex concentrate. Circulation. 2015;131(1):82-90.

49. Majeed A, Agren A, Holmstrom M, Bruzelius M, Chaireti R, Odeberg J, et al. Management of Rivaroxaban- or apixaban-associated major bleeding with prothrombin complex concentrates: a cohort study. Blood. 2017;130(15): 1706-12.

50. collaborators C-t, Shakur H, Roberts I, Bautista R, Caballero J, Coats T, et al. Effects of tranexamic acid on death, vascular occlusive events, and blood transfusion in trauma patients with significant haemorrhage (CRASH-2): a randomised, placebo-controlled trial. Lancet. 2010;376(9734):23-32.

51. Crash-2 Collaborators IBS. Effect of tranexamic acid in traumatic brain injury: a nested randomised, placebo controlled trial (CRASH-2 Intracranial Bleeding Study). BMJ. 2011;343:d3795.

52. Honda Y, Furugohri T, Morishima Y. Tranexamic acid failed to reverse the anticoagulant effect and bleeding by an oral direct factor Xa inhibitor edoxaban. Pharmacology. 2018;101(1-2):92-5.

53. Mayer SA, Brun NC, Begtrup K, Broderick J, Davis S, Diringer MN, et al. Efficacy and safety of recombinant activated factor VII for acute intracerebral hemorrhage. N Engl J Med. 2008;358(20):2127-37.

54. Perzborn E, Heitmeier $\mathrm{S}$, Laux $\mathrm{V}$, Buchmuller A. Reversal of rivaroxabaninduced anticoagulation with prothrombin complex concentrate, activated prothrombin complex concentrate and recombinant activated factor VII in vitro. Thromb Res. 2014;133(4):671-81.

55. Sprugel MI, Kuramatsu JB, Gerner ST, Sembill JA, Beuscher VD, Hagen M, et al. Antiplatelet therapy in primary spontaneous and oral anticoagulationassociated intracerebral hemorrhage. Stroke. 2018;49(11):2621-9.

56. Desborough MJ, Oakland KA, Landoni G, Crivellari M, Doree C, Estcourt L et al. Desmopressin for treatment of platelet dysfunction and reversal of antiplatelet agents: a systematic review and meta-analysis of randomized controlled trials. J Thromb Haemost. 2017;15(2):263-72

\section{Publisher's Note}

Springer Nature remains neutral with regard to jurisdictional claims in published maps and institutional affiliations.

Ready to submit your research? Choose BMC and benefit from:

- fast, convenient online submission

- thorough peer review by experienced researchers in your field

- rapid publication on acceptance

- support for research data, including large and complex data types

- gold Open Access which fosters wider collaboration and increased citations

- maximum visibility for your research: over $100 \mathrm{M}$ website views per year

At BMC, research is always in progress.

Learn more biomedcentral.com/submissions 